\title{
BMJ Open Addressing cognitive impairment following stroke: systematic review and meta-analysis of non-randomised controlled studies of psychological interventions
}

\author{
Niamh A Merriman, ${ }^{1}$ Eithne Sexton, ${ }^{1}$ Grainne McCabe, ${ }^{2}$ Mary E Walsh, ${ }^{1,3}$ \\ Daniela Rohde, ${ }^{\circ}$ Ashleigh Gorman, ${ }^{1}$ Isabelle Jeffares, ${ }^{1}$ Nora-Ann Donnelly, ${ }^{4}$ \\ Niall Pender, ${ }^{5}$ David J Williams, ${ }^{6,7}$ Frances Horgan, ${ }^{3}$ Frank Doyle, ${ }^{1}$ \\ Maev-Ann Wren, ${ }^{4}$ Kathleen E Bennett, ${ }^{1}$ Anne Hickey ${ }^{1}$
}

To cite: Merriman NA, Sexton E, McCabe G, et al. Addressing cognitive impairment following stroke: systematic review and meta-analysis of non-randomised controlled studies of psychological interventions. BMJ Open 2019;9:e024429. doi:10.1136/ bmjopen-2018-024429

- Prepublication history and additional material for this paper are available online. To view these files, please visit the journal online (http://dx.doi. org/10.1136/bmjopen-2018024429).

Received 25 May 2018 Revised 23 October 2018 Accepted 3 January 2019
Check for updates

(C) Author(s) (or their employer(s)) 2019. Re-use permitted under CC BY-NC. No commercial re-use. See rights and permissions. Published by BMJ.

For numbered affiliations see end of article.

Correspondence to Dr Niamh A Merriman; niamhmerriman@rcsi.ie

\section{ABSTRACT}

Objective Cognitive impairment is a pervasive outcome of stroke, reported in over half of patients 6 months poststroke and is associated with increased disability and a poorer quality of life. Despite the prevalence of post-stroke cognitive impairment, the efficacy of existing psychological interventions for the rehabilitation of cognitive impairment following stroke has yet to be established. The aim of this study is to identify psychological interventions from nonrandomised studies that intended to improve post-stroke cognitive function and establish their efficacy.

Design Systematic review and meta-analysis of nonrandomised studies of psychological interventions addressing post-stroke cognitive impairment.

Data sources Electronic searches were performed in the Pubmed, EMBASE and PsycINFO databases, the search dating from inception to February 2017.

Eligibility criteria All non-randomised controlled studies and quasi-randomised controlled trials examining psychological interventions to improve cognitive function following stroke were included, such as feasibility studies, pilot studies, experimental studies, and quasi-experimental studies. The primary outcome was cognitive function. The prespecified secondary outcomes were functional abilities in daily life and quality of life.

Methods The current meta-analyses combined the findings of seven controlled studies, examining the efficacy of psychological interventions compared with treatmentas-usual controls or active controls, and 13 one-group pre-post studies.

Results Results indicated an overall small effect on cognition across the controlled studies (Hedges' $\mathrm{g}=0.38$, $95 \% \mathrm{Cl}=0.06$ to 0.7 ) and a moderate effect on cognition across the one-group pre-post studies (Hedges' $\mathrm{g}=0.51$, $95 \% \mathrm{Cl}=0.3$ to 0.73 ). Specific cognitive domains, such as memory and attention also demonstrated a benefit of psychological interventions.

Conclusions This review provides support for the potential of psychological interventions to improve overall cognitive function post-stroke. Limitations of the study, in
Strengths and limitations of this study

- This systematic review is the first to investigate the effectiveness of psychological interventions aimed at improving general cognitive function post-stroke.

- This systematic review was reported in accordance with the Preferred Reporting Items for Systematic Reviews and Meta-Analyses statement.

- Support for psychological interventions for poststroke cognitive impairment is emergent but further high-quality research is necessary.

- The optimal timing, content, dose, and mode of delivering psychological interventions for post-stroke cognitive impairment is yet to be established.

terms of risk of bias and quality of included studies, and future research directions are explored.

PROSPERO registration number CRD42017069714.

\section{INTRODUCTION}

Stroke is one of the major causes of worldwide death and disability, ${ }^{1}$ with stroke survivors experiencing significant persistent cognitive deficits which impact on functional ability. ${ }^{2}$ Cognitive impairment has been reported in over $50 \%$ of stroke survivors 6 months post-stroke and is associated with a poorer quality of life and increased disability $^{3}$, as well as being related to the presence of depressive symptoms in the longer -term. ${ }^{4}$ Stroke survivors who present with moderate post-stroke cognitive impairment are six times more likely to transition to incident dementia compared with those stroke survivors without cognitive impairment, ${ }^{5}$ with up to $25 \%$ of those with cognitive impairment diagnosed with dementia in 
the 3 years following stroke. ${ }^{6}$ Furthermore, it has been reported that $10 \%$ of stroke survivors develop dementia following a first ever stroke and over 33\% develop dementia following a recurrent stroke. ${ }^{7}$ The consequences of stroke have largely been defined in terms of physical function, which has been the main focus of rehabilitation and research, with evidence demonstrating significant improvements following physical rehabilitation. ${ }^{8}$ However, rehabilitation of post-stroke cognitive impairment has received far less attention. Despite the prevalence of cognitive impairment post-stroke, and the associated poorer outcomes for stroke survivors in terms of physical and psychological well-being, the efficacy of existing psychological interventions for the rehabilitation of cognitive impairment following stroke has yet to be established. ${ }^{10}$

Cognitive rehabilitation has been defined as a 'systematic, functionally oriented service of therapeutic activities that is based on assessment and understanding of the patient's brain-behavioural deficits'. ${ }^{11}$ Five previous Cochrane reviews have examined the effectiveness of post-stroke cognitive rehabilitation. Specifically, these reviews have focused on occupational therapy for cognitive impairment, ${ }^{12}$ memory deficits, ${ }^{13}$ executive dysfunction, ${ }^{14}$ spatial neglect ${ }^{15}$ and attention deficits ${ }^{16}$ following stroke. Similarly, a systematic review was conducted on the effectiveness of neurofeedback as a form of cognitive rehabilitation to improve cognitive function post-stroke. ${ }^{17}$ Each review has concluded that the evidence for the effectiveness of cognitive rehabilitation aimed at each of these domains separately is limited or inconclusive. However, the strict nature of eligibility criteria for inclusion in these reviews resulted in the exclusion of informative non-randomised controlled studies. The pattern of poststroke cognitive impairment suggests that deficits may be distributed across all cognitive domains rather than being confined to one cognitive domain, ${ }^{1819}$ with lesion location predicting the severity of post-stroke cognitive impairment across different cognitive domains. ${ }^{20}{ }^{21}$ Despite the evidence suggesting more dispersed cognitive impairment post-stroke rather than domain-specific deficits, there is, as yet, no review of psychological interventions for post-stroke cognitive impairment that includes the full range of psychological interventions and which target all forms of cognitive impairment (eg, including memory, attention, executive function, etc.). This current review endeavours to capture those non-randomised controlled studies which may be of value when designing a cognitive rehabilitation programme for post-stroke cognitive impairment.

The aim of this systematic review is to identify which types of (non-randomised) psychological interventions have been utilised to improve cognitive function poststroke and to assess the efficacy of these interventions in stroke survivors.

\section{METHODS}

\section{Study design}

This systematic review was conducted in accordance with the Preferred Reporting Items for Systematic Reviews and Meta-Analyses (PRISMA) statement ${ }^{22}{ }^{23}$ (see online supplementary table 1 in appendix A for PRISMA checklist). The review protocol was registered with the International Prospective Register of Systematic Reviews (PROSPERO) on 30 June 2017, and the full protocol was published. $^{10}$

\section{Eligibility criteria}

Types of study

All non-randomised controlled studies and quasi-randomised controlled trials examining psychological interventions with a sample size $\geq 2$ aimed at improving cognitive function following stroke were included in this systematic review. These included feasibility studies, pilot studies, experimental studies and quasi-experimental studies. Randomised controlled trials, review articles, letters, editorials, qualitative studies, case studies with a sample size of 1 , animal studies, and study protocols were excluded.

\section{Participants}

Studies of an adult population (age 18+) were included. Studies of participants with mixed aetiologies (eg, traumatic brain injury/stroke mix) were excluded unless data were available, or made available on contacting the study authors, for those participants with a primary diagnosis of stroke (ischaemic, intracranial haemorrhagic, subarachnoid haemorrhage) or if the study had more than $75 \%$ of people with stroke in the study sample. ${ }^{16}$

\section{Types of interventions}

Given the wide variation in types of interventions to address post-stroke cognitive impairment, psychological interventions of any type and duration intended to rehabilitate cognition post-stroke were included. Examples of the eligible interventions included: neuropsychological interventions; computerised interventions; cognitive and/or behavioural interventions, including problem solving; strategy training (eg, mnemonic strategies, mental imagery); goal management training; and self-efficacy training. Studies with a focus on poststroke cognition outcomes of pharmacological or other non-psychological interventions were excluded.

\section{Comparisons or control}

Studies addressing psychological interventions to improve cognition following stroke in comparison to a usual/routine care (eg, no contact, wait-list) control arm or an active control arm were included. Active controls included sham (placebo) or non-specific computerised cognitive training or low-dose training.

\section{Outcome measures}

Cognitive function from pre-intervention to post-intervention was the primary outcome of interest, with cognitive 
function assessed using a validated measure of domain specific cognitive function, including those comprised in the National Institute of Neurological Disorders and Stroke (NINDS) $30 \mathrm{~min}$ or $60 \mathrm{~min}$ battery of cognitive assessment. ${ }^{24}$ As a number of studies report scores from cognitive screening tools such as the Montreal Cognitive Assessment (MoCA) ${ }^{25}$ Mini-Mental State Examination (MMSE),${ }^{26}$ Loewenstein Occupational Therapy Cognitive Assessment (LOTCA) battery ${ }^{27}$ and the Cognistat Assessment, ${ }^{28}$ these validated measures of cognition were also acceptable. Other validated measures of domain specific cognitive function were also acceptable, as were validated measures of subjective cognitive function (eg, Multifactorial Memory Questionnaire ${ }^{29}$ ). All eligible outcomes per study and cognitive domain were included. Index scores were not included if the individual domain scores were available.

Secondary outcomes of interest included reports of functional abilities in daily life and quality of life, including activities of daily living (ADL), for example using the Functional Independence Measure ${ }^{30}$; Instrumental ADL (IADL), for example using the Nottingham Extended ADL (NEADL) scale ${ }^{31}$; Quality of life (QoL), based on stroke-specific or generic QoL assessment measures.

\section{Search strategy for the identification of relevant studies}

The search strategy was developed in collaboration with a subject librarian. Three databases covering the medical and psychological peer-reviewed literature were searched: Pubmed (http://www.ncbi.nlm.nih. gov/pubmed/), EMBASE (https://www.embase.com), and PsycINFO (http://www.apa.org/pubs/databases/ psycinfo/index.aspx). The Pubmed search strategy is detailed in online supplementary table 1 in appendix B. These terms were also mapped to Medical Subject Heading terms and similar terms in EMBASE and PsycINFO, and the search carried out dating from inception to February 2017. The search was restricted to articles published in English.

Searches were exported to EndNote X7 to build a master file of all references. In addition to the database searches, the reference list of included articles was reviewed for relevant studies and previous reviews. A citation search was also carried out to identify papers cited, using Web of Science. A hand search was conducted of the four journals that generated the greatest number of relevant articles.

\section{Screening of the studies}

Duplicates were identified using EndNote X7 'find duplicates' function. Titles and abstracts were assessed for eligibility by one reviewer (NAM). A random sample of $85 \%$ was independently double screened between four second reviewers (MEW, IJ, AG and DR). The full texts of papers identified as potentially eligible were obtained for review by two reviewers (NAM and $\mathrm{AH}$ ), with differences resolved through discussion.

\section{Data extraction}

Data from included studies were extracted using a standardised form, including: authors, study design, sample size (baseline and follow-up), sample description, target population characteristics, intervention type, intervention content, control (passive, active), length of follow-up, type of outcome, primary and secondary outcomes (listed above), comments and study conclusions. Study authors were contacted for missing data or further information if necessary. Coding of outcome measures into cognitive domains was done by NAM and was approved by an expert in clinical neuropsychology (NP; see online supplementary table 1 in appendix $\mathrm{C}$ for categorisation of cognitive measures into cognitive domains). Data from most studies were recorded as mean and SD for each group at baseline and post-intervention and/or follow-up, or estimated from the median, range, and sample size where the mean and SDs were not available, using standard approaches. ${ }^{32}$ In two instances, t-test and $p$ value, ${ }^{33}$ and $\mathrm{F}$ value and $\mathrm{p}$ value ${ }^{34}$ were recorded and the standardised mean difference (SMD) was calculated from the statistics.

When studies presented data comparing stroke patients' performance with that of healthy adults, or with those with cognitive impairment resulting from aetiology other than stroke, only data from stroke patients were used and analysed. Where studies presented data from multiple intervention groups, all relevant intervention groups were combined into a single group, and all relevant control groups were combined into a single control group. ${ }^{35}$

\section{Risk of bias}

Two authors (NAM, ES) independently assessed the strengths and weaknesses of each eligible study using the risk of bias in non-randomised studies - of Interventions (ROBINS-I) tool by the Cochrane Collaboration, ${ }^{36}$ with disagreements resolved through discussion. As all of the included studies were non-randomised or quasi-randomised controlled studies, the minimum risk of bias possible was moderate risk. Those studies without a control group were considered to have a critical risk of bias by virtue of the lack of a comparison group. No study was excluded as a result of findings from the risk of bias assessments, but instead controlled studies and one-group pre-post studies were analysed separately. Publication bias was assessed by visually inspecting funnel plots for asymmetry. ${ }^{37}$

\section{Quality of evidence}

Two authors (NAM, ES) assessed the overall quality of the evidence of the studies using the Grading of Recommendations Assessment, Development and Evaluation (GRADE) tool. ${ }^{38}$ As all included studies were non-randomised controlled studies or quasi-randomised controlled studies, they were automatically assigned a baseline rating of low quality. Studies had potential to be upgraded if there was a large effect size, evidence of a dose-response relationship, or when all possible confounders would have reduced the observed effect. Similarly, studies could be downgraded if there were 
serious concerns relating to risk of bias, inconsistency of results, indirectness of outcome measure, imprecision of the effect estimate or publication bias. ${ }^{38}$ The quality of the studies was judged as high (further research is very unlikely to change the confidence in the effect estimates), moderate (further research is likely to have an important impact on the confidence in the effect and may change the estimate), low (further research is very likely to have an important impact on the confidence in the effect and is likely to change the estimate) and very low (any estimate of the effect is very uncertain). ${ }^{38}$

\section{Strategy for data synthesis}

The primary outcome was SMD, calculated as Hedges' $\mathrm{g},{ }^{39}$ and with precision estimated by the $95 \%$ CI of change in outcome measures between the intervention and control groups for the controlled studies from pre- to post-intervention and follow-up. For the one-group prepost studies, SMD and $95 \%$ CI were calculated as change in outcome measures from pre-intervention to post-intervention and followed up using Becker's formula, ${ }^{40}$ where the correlation between pre-outcome and post-outcome measures was estimated at 0.5. A positive SMD is indicative of a beneficial effect of the intervention over the control, or better performance post-intervention compared with pre-intervention in the case of the one-group studies. Hedges' $g$ estimates are comparable to Cohen's $d^{41}-$ 'trivial' (Effect Size $(\mathrm{ES})<0.20$ ), 'small' (ES $\geq 0.20<0.50$ ), 'moderate' (ES $\geq 0.50<0.80)$ or 'large' (ES $\geq 0.80$ ). Pooling of standardised mean effects across studies was performed using a random effects model. Analyses were performed for overall cognitive outcomes, as well as for each cognitive or behavioural domain separately. When studies presented data from more than one domain per outcome for analysis, results were combined to produce a single study level SMD, with the mean estimate and SE of the related tests, as per established procedure. ${ }^{42-44}$

Heterogeneity was quantified using the $\mathrm{I}^{2}$ statistic - low $(25 \%)$, moderate $(50 \%)$ or large $(75 \%) .{ }^{45}$ Funnel plots were inspected to identify outliers as potential sources of heterogeneity. A planned series of sub-group analyses based on intervention design features and population characteristics $^{10}$ was not performed due to very low to null heterogeneity among study level cognitive outcomes following random effects analysis for both the controlled group design studies $\left(I^{2}=9.7 \%\right)$ and the one-group pre-post design studies $\left(\mathrm{I}^{2}=0 \%\right)$, respectively. All analyses were performed using Stata version 14.

\section{Patient and public involvement}

Patients were not involved in the design of this systematic review of secondary data.

\section{RESULTS}

\section{Study selection}

The searches returned 19843 records, with 2613 duplicates, resulting in 17230 titles and abstracts screened for inclusion. Following title and abstract screening, 251

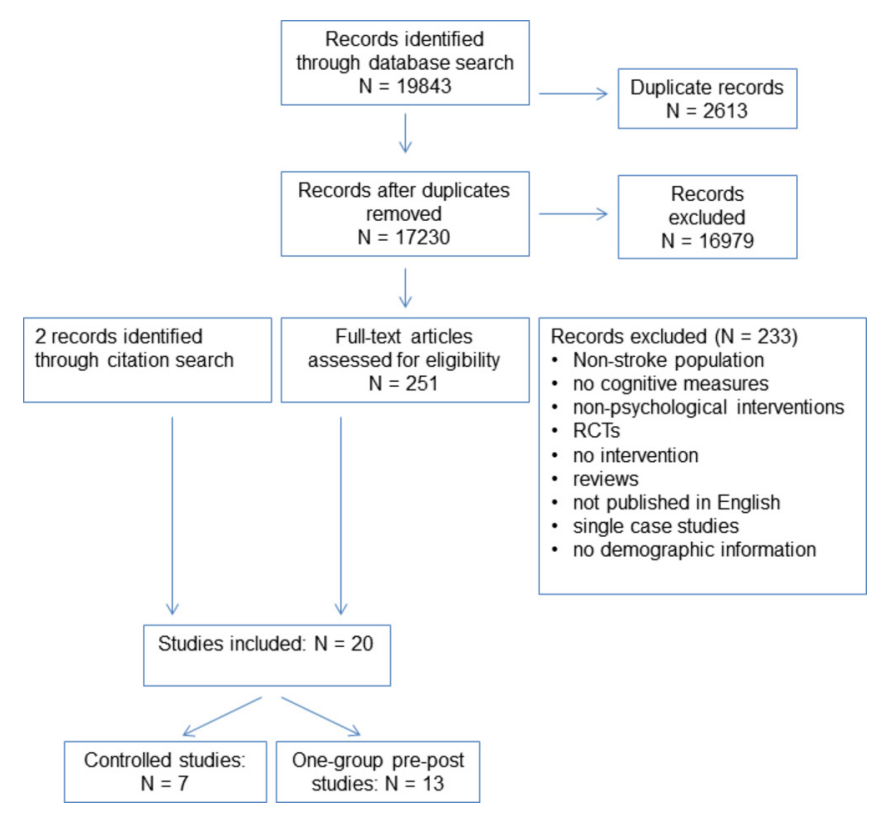

Figure 1 Reporting Items for Systematic Reviews and Meta-Analyses flow chart of included studies. RCT, randomised controlled trial.

papers were identified for full text screening. Subsequent to the full text screen, 233 papers were excluded; reasons for exclusion are detailed in PRISMA flowchart in figure 1. Two additional full texts were identified following a citation search, resulting in 20 included studies (figure 1).

\section{Characteristics of included studies}

Controlled studies

The seven included studies comprised four quasi-randomised studies and three controlled studies, with 201 total participants (intervention: $\mathrm{n}=78$, mean group size: $\mathrm{n}=11$ [SD=7.71]; control: $\mathrm{n}=123$, mean group size: $\mathrm{n}=11$ $[\mathrm{SD}=5.88])$ and reported 64 cognitive/behavioural outcomes which met the inclusion criteria for this review. Mean age across studies was 55.4 years $(\mathrm{SD}=6.0)$, and $39 \%$ of participants were female. All but one study ${ }^{34}$ reported sex ratios of participants. The mean time in months since stroke onset was $14.2(\mathrm{SD}=20.2)$. The mean reported years of education reported in three of the studies ${ }^{33} 346$ was 13.3 years $(\mathrm{SD}=2.0)$. Across the seven studies, $11.9 \%$ of patients suffered a haemorrhagic stroke, $21.9 \%$ suffered an ischaemic stroke, $3.5 \%$ experienced a subarachnoid haemorrhage, and $53.7 \%$ had unspecified stroke aetiology (see online supplementary table 1 in appendix D).

In terms of the cognitive domain each intervention was aiming to target, three of the interventions targeted attention, ${ }^{344748}$ two addressed memory, ${ }^{3349}$ and two aimed to improve executive function. ${ }^{46}{ }^{50}$ Both of the interventions targeting memory were delivered in group format in a hospital setting ${ }^{33} 49$ while the other interventions were delivered on an individual basis either in a hospital setting, ${ }^{34}$ in the participant's home,${ }^{46}{ }^{50}$ or in a research laboratory. ${ }^{47} 48$ Three interventions comprised computerised cognitive training and were either unsupervised ${ }^{50}$ or it was not reported who supervised the intervention. ${ }^{47} 48 \mathrm{An}$ 
occupational therapist oversaw the intervention delivery in three studies, ${ }^{34} 4649$ which comprised pen and paper and strategy-based tasks (individual or group setting, respectively), while a neuropsychologist supervised the other group-based study which also used strategy-based tasks. ${ }^{33}$ The average number of intervention sessions was $15.7(\mathrm{SD}=5.1)$ with mean session length of $68.3 \mathrm{~min}$ $(\mathrm{SD}=52.2)$. The average intervention dose was 14.3 hours $(\mathrm{SD}=5.3)$.

Six of the seven studies were rated both at the study level and at cognitive domain outcome level as having a serious risk of bias as measured by the ROBINS-I tool, ${ }^{33}{ }^{46-50}$ with one study rated as having a moderate risk of bias at the study level ${ }^{34}$ (online supplementary table 1 in appendix E). Based on the GRADE approach, the overall quality of the evidence was rated as very low, with no studies being upgraded from their initial rating, and all seven studies downgraded due to either serious risk of bias and/or imprecise effect estimates based on unadjusted or minimally adjusted analyses (online supplementary table 1 in appendix F).

\section{One group pre-post studies}

The 13 studies comprised eight one-group pre-post studies, three case-control pre-post studies, and two single case-studies series, both with a sample size of $>2$ stroke patients. Two one-group pre-post studies and one single case-study series reported baseline data collected 2 weeks prior to the pre-intervention assessment. ${ }^{51-53}$ The total number of participants was 103 , with a mean group size of $8.1 \quad(\mathrm{SD}=6.2)$ and reported a combined total of 115 cognitive/behavioural outcomes which met the inclusion criteria for this review. Mean age across studies was 62.6 years $(\mathrm{SD}=8.54)$, and $40 \%$ of participants were female. Three studies ${ }^{515455}$ did not report sex ratios of participants, one study did not report participant ages ${ }^{56}$ and three did not report time since stroke onset. ${ }^{525758}$ The mean time in months since stroke onset was $23.9(\mathrm{SD}=32.5)$. The mean number of years of education reported in three of the studies ${ }^{56}{ }^{57}{ }^{59-61}$ was 13.1 years $(\mathrm{SD}=2.7)$. Across the 13 studies, $1.9 \%$ of patients suffered a haemorrhagic stroke, $21.9 \%$ suffered an ischaemic stroke, $6.7 \%$ experienced a subarachnoid haemorrhage, and $69.5 \%$ had unspecified stroke aetiology (see online supplementary table 1 in appendix D).

Three of the studies targeted memory, ${ }^{52} 5459$ two addressed memory and attention together, ${ }^{55} 60$ three targeted attention ${ }^{5161}$ with one of these studies combining attention and ADL training. ${ }^{62}$ Four studies focused on improvement in general cognitive function alone ${ }^{57}$ or in combination with ADL training, ${ }^{56} 5863$ and one study aimed to improve executive function. ${ }^{53}$ Eight studies comprised computerised cognitive training and were supervised in either a hospital setting by an occupational therapist, ${ }^{54}$ were supervised by neuropsychologists or NP Master's students and delivered in the home or university, ${ }^{57} 5960$ were supervised by the study researchers in a community setting or unreported setting, ${ }^{55} 56$ or the study did not report if and by whom the intervention was supervised. ${ }^{5152}$ One intervention targeting general cognitive function and ADL was delivered in a hospital-based group format by an occupational therapist, ${ }^{58}$ while the remaining interventions delivered under the supervision of an occupational therapist on an individual basis were either home-based recreational games for cognitive remediation $^{63}$ or hospital-based mental imagery training. ${ }^{62} \mathrm{~A}$ psychologist and therapist supervised a further mental imagery and pen-and-paper based intervention, respectively, in unspecified settings. ${ }^{5361}$ The average number of intervention sessions was $29.6(\mathrm{SD}=42.8)$ with mean session length of $51.7 \mathrm{~min}(\mathrm{SD}=17.3)$. The average intervention dose was 27.8 hours $(\mathrm{SD}=43.5)$.

All 13 studies were rated both at the study level and at cognitive domain outcome level as having a critical risk of bias as measured by the ROBINS-I tool (online supplementary table 2 in appendix E), by virtue of lack of a control group. Similarly the overall quality of the evidence was rated as very low, based on the GRADE approach, with no studies being upgraded from their initial rating, and all 13 studies inevitably downgraded due to critical risk of bias because of the lack of a control group.

\section{Meta-analysis of controlled studies' outcomes Overall efficacy on cognitive outcomes}

The overall effect of psychological interventions on cognition was small and statistically significant ( $\mathrm{k}=7$, Hedges' $\mathrm{g}=0.38,95 \% \mathrm{CI}=0.06$ to 0.7$)$ and had a low level of heterogeneity $\left(\mathrm{I}^{2}=9.7 \%, \mathrm{p}=0.36\right)$ (figure 2 ).

Although asymmetry was not formally assessed due to the small number of included studies, the funnel plot revealed one outlier, ${ }^{48}$ which reported a large SMD $(=2.27)$. Removal of this study reduced heterogeneity to zero $\left(I^{2}=0 \%, p=0.93\right)$ and the summary effect was also reduced ( $\mathrm{k}=6$, Hedges' $\mathrm{g}=0.3,95 \% \mathrm{CI}=0.00$ to 0.6 ) (see online supplementary figures $1 \mathrm{a}$ and $\mathrm{b}$ in appendix $\mathrm{G}$ ).

\section{Domain-specific efficacy}

For overall estimates of summary effects, see table 1 .

\section{Attention}

The effect size of psychological interventions on attention was large and statistically significant ( $k=3$, Hedges' $\mathrm{g}=0.98,95 \% \mathrm{CI}=0.24$ to 1.72$)$ and a moderate level of heterogeneity $\left(\mathrm{I}^{2}=40.3 \%, \mathrm{p}=0.19\right)$ (see online supplementary figure 2 in appendix $G)$.

Again, the funnel plot indicated an outlier ${ }^{48}$ with a large SMD (>2.0). Removal of this study reduced heterogeneity to zero $\left(\mathrm{I}^{2}=0 \%, \mathrm{p}=0.58\right)$ and the summary effect was also reduced ( $\mathrm{k}=2$, Hedges' $\mathrm{g}=0.72,95 \% \mathrm{CI}=0.15$ to 1.29 ) (see online supplementary figures $3 a$ and $b$ in appendix $G$ ).

\section{Other domains}

Statistically non-significant results were found for the following domains: executive function $(\mathrm{k}=3)$; processing speed ( $k=3)$; memory $(k=2)$; and IQ ( $k=2)$. Meta-analyses of outcomes of subjective memory, ${ }^{3}$ perceptual reasoning, ${ }^{34}$ subjective executive function, self-efficacy, 
Study

ID

$\mathrm{ES}(95 \% \mathrm{Cl})$

$\%$

Weight

\begin{tabular}{|c|c|c|}
\hline & & \\
\hline Poulin (2017) & $-0.07(-1.27,1.12)$ & 6.75 \\
\hline Lindelov (2016) & $0.17(-0.76,1.11)$ & 10.79 \\
\hline Miller (2014) & $0.32(-0.36,1.00)$ & 19.16 \\
\hline Hildebrandt (2006) & $0.21(-0.30,0.71)$ & 31.02 \\
\hline Sturm (2004) & $2.27(0.63,3.91)$ & 3.69 \\
\hline Sturm (1997) & $0.56(-0.24,1.36)$ & 14.22 \\
\hline Young (1983) & $0.53(-0.27,1.33)$ & 14.37 \\
\hline Overall (l-squared $=9.7 \%, p=0.355$ ) & $0.38(0.06,0.70)$ & 100.00 \\
\hline NOTE: Weights are from random effects analysis & & \\
\hline $\begin{array}{c}1 \\
-3.91\end{array}$ & $\begin{array}{l}T \\
91\end{array}$ & \\
\hline
\end{tabular}

Figure 2 Forest plot of included controlled studies at study level.

and instruments and $\mathrm{ADL}^{46}$ were not performed as only one study for each domain was available for analysis.

\section{Meta-analysis of outcomes of one group pre-post studies}

Overall efficacy on cognitive outcomes

The overall effect of psychological interventions on cognition across the one-group pre-post studies was moderate and statistically significant ( $\mathrm{k}=13$, Hedges' $\mathrm{g}=0.51,95 \% \mathrm{CI}=0.3$ to 0.73 ) (figure 3 ). Visual inspection of the funnel plot revealed potential asymmetry. It is important to note that asymmetry in funnel plots may be the result of a number of causes, including heterogeneity or chance. ${ }^{37}$ However, there was null heterogeneity across studies $\left(\mathrm{I}^{2}=0 \%, \mathrm{p}=0.8\right)$.

Domain-specific efficacy

For overall estimates of summary effects, see table 1 .

Memory

The effect size of psychological interventions on memory was moderate and statistically significant ( $k=7$, Hedges' $\mathrm{g}=0.4,95 \% \mathrm{CI}=0.13$ to 0.67 ) with null heterogeneity

\begin{tabular}{|c|c|c|c|c|c|}
\hline Domain & Studies & SE & $95 \mathrm{Cl}$ Lower & $95 \mathrm{Cl}$ Higher & $I^{2}$ \\
\hline \multicolumn{6}{|l|}{ Controlled studies } \\
\hline Executive function & $k=3$ & 0.14 & -0.27 & 0.56 & 0.00 \\
\hline Processing speed & $k=3$ & 0.34 & -0.05 & 0.72 & 0.00 \\
\hline Attention & $k=3$ & 0.98 & 0.24 & 1.72 & 40.3 \\
\hline Memory & $k=2$ & 0.27 & -0.14 & 0.67 & 0.00 \\
\hline IQ & $k=2$ & 0.30 & -0.59 & 1.19 & 52.6 \\
\hline \multicolumn{6}{|l|}{ One-group pre-post studies } \\
\hline Memory & $k=7$ & 0.40 & 0.13 & 0.67 & 0.00 \\
\hline Attention & $k=5$ & 0.52 & 0.02 & 1.01 & 0.00 \\
\hline Executive function & $k=5$ & 0.12 & -0.12 & 0.35 & 0.00 \\
\hline Global cognition & $k=4$ & 0.90 & 0.38 & 1.41 & 19.5 \\
\hline Instruments and activities of daily living & $k=4$ & 0.26 & -0.31 & 0.82 & 0.00 \\
\hline Processing speed & $k=3$ & 0.57 & -0.07 & 1.21 & 71.0 \\
\hline IQ & $k=3$ & 0.49 & -0.27 & 1.25 & 39.4 \\
\hline Subjective memory & $k=2$ & -0.21 & -0.84 & 0.43 & 61.3 \\
\hline Perceptual reasoning & $k=2$ & 0.59 & -0.17 & 1.35 & 67.3 \\
\hline Quality of life & $k=2$ & 0.61 & -0.33 & 1.55 & 0.00 \\
\hline
\end{tabular}


Study

ID
$\%$

Weight

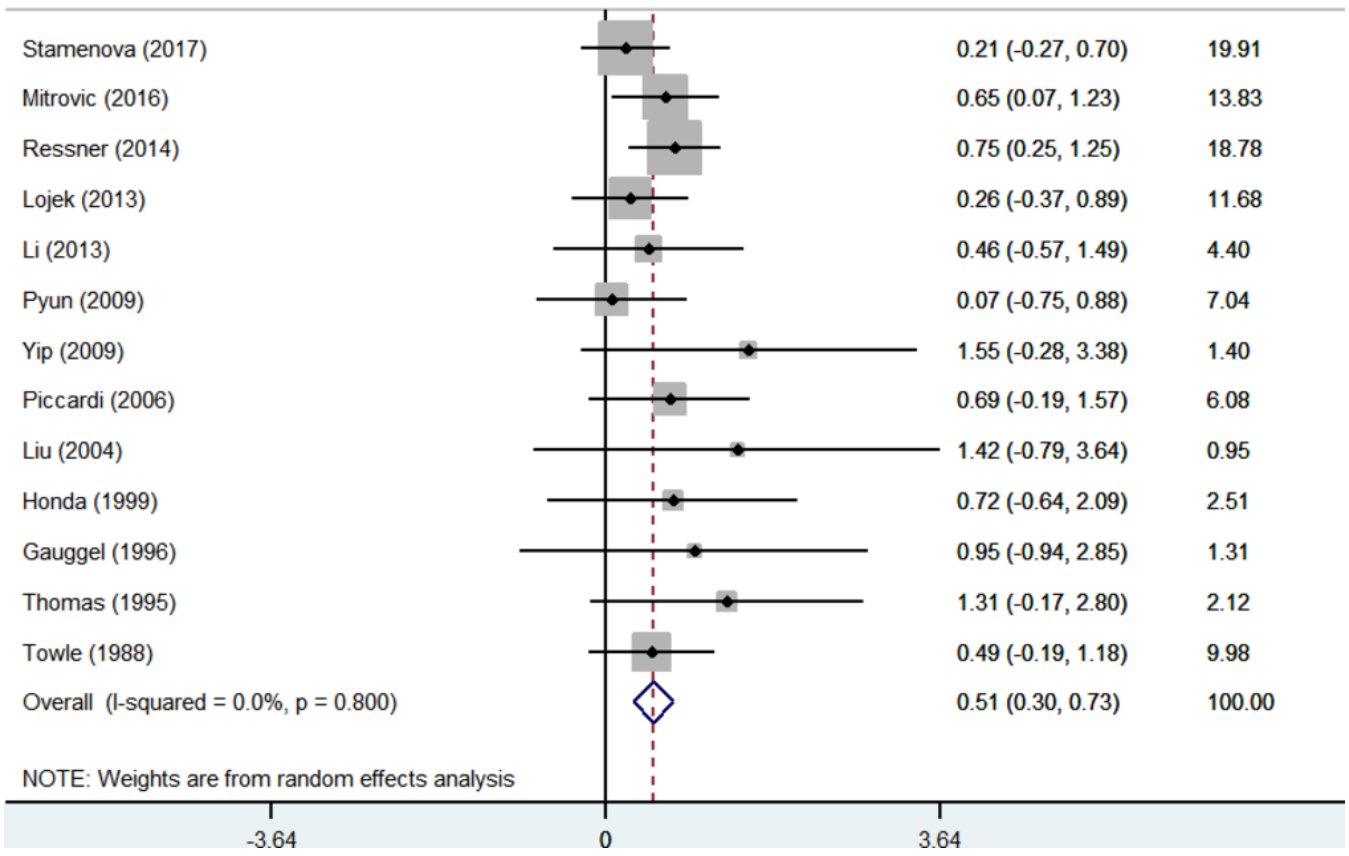

Figure 3 Forest plot of included one-group pre-post studies at study level.

$\left(\mathrm{I}^{2}=0 \%, \mathrm{p}=0.53\right) \quad$ (see online supplementary figure 4 in appendix G). Although asymmetry was not formally assessed due to the small number of included studies, the funnel plot revealed one outlier, ${ }^{51}$ which reported a large SMD (=1.70). Removal of this study reduced the summary effect slightly ( $\mathrm{k}=6$, Hedges' $\mathrm{g}=0.38,95 \% \mathrm{CI}=0.11$ to 0.65 ) (see online supplementary figures $5 \mathrm{a}$ and $\mathrm{b}$ in appendix G).

\section{Attention}

The attention effect size was also moderate and statistically significant ( $\mathrm{k}=5$, Hedges' $\mathrm{g}=0.52,95 \% \mathrm{CI}=0.02$ to 1.01 ) with null heterogeneity $\left(\mathrm{I}^{2}=0 \%, \mathrm{p}=0.47\right.$ ) (see online supplementary figure 6 in appendix G). However, the funnel plot indicated two outliers ${ }^{51} 62$ with a large SMD (both >1.14). Removal of these studies reduced the summary effect to small and non-significant ( $k=3$, Hedges' $\mathrm{g}=0.41,95 \% \mathrm{CI}=-0.11$ to 0.93$)$, with no effect on heterogeneity $\left(\mathrm{I}^{2}=0 \%, \mathrm{p}=0.64\right)$ (online supplementary figures $7 \mathrm{a}$ and $\mathrm{b}$ in appendix $\mathrm{G})$.

\section{Global cognition}

The global cognition effect size was large and statistically significant ( $k=4$, Hedges' $\mathrm{g}=0.9,95 \% \mathrm{CI}=0.38$ to 1.41$)$ with a low level of heterogeneity $\left(\mathrm{I}^{2}=19.5 \%, \mathrm{p}=0.29\right)$ (online supplementary figure 8 in appendix G). However, all studies included in this analysis, with the exception of one, ${ }^{63}$ had an effect size $\geq 1$ which drove the summary effect.

\section{Other domains}

Statistically non-significant results were found for the following domains: executive function $(\mathrm{k}=5)$; instruments and ADL $(\mathrm{k}=4)$; processing speed $(\mathrm{k}=3) ; \mathrm{IQ}(\mathrm{k}=3)$; subjective memory $(\mathrm{k}=2)$; perceptual reasoning $(\mathrm{k}=2)$ and quality of life $(\mathrm{k}=2)$. Meta-analyses of outcomes of subjective executive function, ${ }^{53}$ self-efficacy ${ }^{56}$ and language comprehension $^{57}$ were not performed as only one study for each domain was available for analysis.

\section{Long-term outcomes}

Only one controlled study reported 1 month follow-up data beyond the post-intervention assessment for both intervention and control group. ${ }^{46}$ Similarly two one-group pre-post studies reported outcomes at 2 weeks and 4 weeks following the postintervention assessment, respectively. ${ }^{52} 53$ Therefore, it was not possible to pool data due to the insufficient number of studies.

\section{DISCUSSION}

Results from this systematic review suggest that psychological interventions may be effective for improving overall cognitive function post-stroke, with the specific cognitive domains of attention and memory demonstrating a greater benefit than other cognitive domains, for example, executive function. However, it is imperative to acknowledge the low quality and high risk of bias of the included studies. Furthermore, due to an insufficient number of studies, it was not possible to ascertain the effectiveness of psychological interventions for domains such as subjective memory, subjective executive function, self-efficacy, instruments and ADL, perceptual reasoning, or language comprehension. Post-stroke cognitive impairment is ubiquitous and has been shown to have negative implications for quality of life and functional 
ability. ${ }^{3}$ Similarly, cognitive impairment following stroke may also impact negatively on the stroke patients' ability to participate fully in their rehabilitation and reach their full rehabilitation potential due to an inability to concentrate efficiently, encode/recall information, or plan and sequence their behaviour. ${ }^{64}$

The effect size for overall cognition was small and significant for meta-analysis of controlled studies, and moderate for one-group pre-post studies. The effect size for attention was quite large in the controlled studies and was driven by a study with an SMD of $>2$. The most recent Cochrane review of cognitive rehabilitation for attention deficits following stroke reported a significant moderate effect of intervention on divided attention, though no evidence to support the effectiveness of cognitive rehabilitation for improvement of global attention. ${ }^{16}$ Due to the small number of included studies assessing attentional function, it was not possible to examine the effect of psychological interventions on sub-domains of attention.

The meta-analyses of individual domains across one-group pre-post studies revealed moderate to large effect sizes for memory, attention and global cognition. It is important to note that the global cognition domain comprised scores from cognitive screening tools as a measure of global cognition, such as the MMSE, ${ }^{26}$ the LOTCA $^{27}$ and the Cognistat ${ }^{28}$ rather than scores from comprehensive cognitive assessments. Interestingly, none of the included studies used the MoCA. ${ }^{25}$ A recent systematic review found that the MMSE is the most widely used cognitive screening tool (similarly in the current review), despite its lack of criterion validity, whereas the MoCA has good predictive validity in assessing overall cognition post-stroke. ${ }^{65}$

The current findings of significant effects for memory contrast with the Cochrane review of cognitive rehabilitation for memory deficits following stroke, which found small significant intervention effects on subjective memory but not objective measures of memory. Although the current review has found evidence of psychological interventions for post-stroke cognitive impairment being most effective in the domains of attention and memory, it is worth noting that studies with a high risk of bias, such as those included in this review, have been found to overestimate effect sizes. ${ }^{66}$ Additionally, due to the lack of follow-up assessment data, it is unknown whether the significant effects revealed by the meta-analyses would endure over time.

Interestingly, no benefit of psychological interventions for executive function was found in the meta-analysis for either study design. Previous systematic reviews have also failed to find evidence of the efficacy of interventions aimed to improve executive function, due to the lack of high quality studies,${ }^{14}$ and also due to a dearth of studies aiming to improve executive function in the acute stroke phase, despite the potential benefit this may have on rehabilitation participation. ${ }^{67}$ Moreover, considering that executive dysfunction is thought to be a predictor of future dementia and disability in a stroke population, ${ }^{68} 69$ future studies should endeavour to include more executive function training. In this systematic review and meta-analyses, typically small sample sizes were employed across studies specifically training executive function. Thus, they were not adequately powered to detect changes across all of the relevant cognitive domains, resulting in a number of individual studies demonstrating no significant effects. ${ }^{460}$

The overall quality of the included studies, based on the GRADE approach, was very low. This was due in most part to the serious and critical risk of bias inherent in non-randomised controlled studies encompassing controlled studies and one-group pre-post studies, respectively. The included studies described interventions, which were complex in nature, and varied across the cognitive domain they aimed to target, the medium through which the intervention was delivered, the healthcare professional who facilitated the intervention delivery, and the length, dose, and duration of interventions. However, these methodological differences across studies did not result in statistically significant heterogeneity and thus did not warrant secondary analyses comparing subgroups of participants or types of interventions. Moreover, the relatively small number of included studies and the small number of participants across controlled studies and one-group pre-post studies, respectively, also limited our ability to perform secondary analyses if statistical heterogeneity had been present. Although pooled effect sizes were calculated by aggregating the standardised mean difference at the study level in order to control for the diversity of study outcome measures and intervention components, it is possible that these differences across studies could have contributed to the variability of the study findings. Therefore, based on our analysis and the diversity of study characteristics, it was not possible to ascertain which type of psychological intervention is most beneficial for addressing post-stroke cognitive impairment. Although the cognitive domains of attention and memory appear to benefit most from intervention, it is necessary to consider the poor quality and high risk of bias of the evidence.

Methodological quality was very low in the included studies due to the inherent high risk of bias of including non-randomised controlled studies. However, it is important to establish interventions that address the psychological and cognitive sequelae of stroke. Future randomised controlled studies should be designed and adequately powered to evaluate potential to improve cognitive function post-stroke, with an emphasis on improving executive function. Furthermore, adequate description of intervention design and implementation should be provided in future studies in order to maximise replication across diverse settings using templates such as the Template for Intervention Description and Replication checklist. ${ }^{70}$ They should also include follow-up assessment time points to determine the long-term effects of any benefit on cognition.

The pattern of post-stroke cognitive impairment is diffuse and rarely confined to one specific cognitive 
domain. ${ }^{18} 19$ This is the first single review to investigate non-randomised controlled studies of the effectiveness of psychological interventions aimed at improving general cognitive function across multiple cognitive domains poststroke. The results of this review add to the evidence base for establishing the effectiveness of psychological interventions for post-stroke cognitive impairment, while highlighting the diversity of outcome measures and intervention components utilised across studies. This review will inform the development of a cognitive rehabilitation programme as part of a wider study, the StrokeCog study, ${ }^{71}$ aimed at improving cognitive function post-stroke.

\section{Author affiliations}

${ }^{1}$ Division of Population Health Sciences, Royal College of Surgeons in Ireland, Dublin, Ireland

${ }^{2}$ Library, Royal College of Surgeons in Ireland, Dublin, Ireland

${ }^{3}$ School of Physiotherapy, Royal College of Surgeons in Ireland, Dublin, Ireland ${ }^{4}$ Social Research Division, Economic and Social Research Institute, Dublin, Ireland ${ }^{5}$ Department of Psychology, Beaumont Hospital, Dublin, Ireland

${ }^{6}$ Department of Geriatric and Stroke Medicine, Beaumont Hospital, Dublin, Ireland ${ }^{7}$ Department of Geriatric and Stroke Medicine, Royal College of Surgeons in Ireland, Dublin, Ireland

Contributors NAM, ES, N-AD, GMC, NP, DR, IJ, AG, MEW, DW, FD, FH, M-AW, KEB and $\mathrm{AH}$ contributed to the conception and design of the study, the development of the search strategy, the establishment of the inclusion and exclusion criteria, data extraction criteria, analyses and interpretation. NAM, DR, IJ, AG and MEW performed the study search, screening and extraction of data. NAM drafted the manuscript, and $A H, K E B, D W, N P, F H$ and FD provided critical revision of the paper. All authors read and approved the final manuscript.

Funding This research was funded by the Health Research Board of Ireland Interdisciplinary Capacity Enhancement (ICE) award (2016-19): The StrokeCog study: modelling and modifying the consequences of stroke-related cognitive impairment through intervention (Grant code: ICE-2015-1048).

\section{Competing interests None declared.}

Patient consent for publication Not required.

Ethics approval We did not seek formal ethical approval for this study as primary data were not collected.

Provenance and peer review Not commissioned; externally peer reviewed.

Data sharing statement We, authors, agree that, should the article be accepted, the BMJ Open shall take over the authors' rights relating to this article, which shall become the property of the journal.

Open access This is an open access article distributed in accordance with the Creative Commons Attribution Non Commercial (CC BY-NC 4.0) license, which permits others to distribute, remix, adapt, build upon this work non-commercially, and license their derivative works on different terms, provided the original work is properly cited, appropriate credit is given, any changes made indicated, and the use is non-commercial. See: http://creativecommons.org/licenses/by-nc/4.0/.

\section{REFERENCES}

1. Feigin VL, Norrving B, Mensah GA. Global burden of stroke. Circ Res 2017;120:439-48.

2. Tatemichi TK, Desmond DW, Stern Y, et al. Cognitive impairment after stroke: frequency, patterns, and relationship to functional abilities. J Neurol Neurosurg Psychiatry 1994;57:202-7.

3. Mellon L, Brewer L, Hall P, et al. Cognitive impairment six months after ischaemic stroke: a profile from the ASPIRE-S study. BMC Neurol 2015;15:1-9.

4. Nys GM, van Zandvoort MJ, van der Worp HB, et al. Early cognitive impairment predicts long-term depressive symptoms and quality of life after stroke. J Neurol Sci 2006;247:149-56.

5. Narasimhalu K, Ang S, De Silva DA, et al. Severity of CIND and $\mathrm{MCl}$ predict incidence of dementia in an ischemic stroke cohort. Neurology 2009;73:1866-72.
6. Sachdev PS, Chen X, Brodaty $\mathrm{H}$, et al. The determinants and longitudinal course of post-stroke mild cognitive impairment. $J$ Int Neuropsychol Soc 2009;15:915-23.

7. Pendlebury ST, Rothwell PM. Prevalence, incidence, and factors associated with pre-stroke and post-stroke dementia: a systematic review and meta-analysis. Lancet Neurol 2009;8:1006-18.

8. Horgan F, Hickey A, McGee H, et al. National Audit of Stroke Care [Internet], Dublin, Ireland. 2008 http://epubs.rcsi.ie/psycholrep/17/.

9. Saka O, McGuire A, Wolfe C. Cost of stroke in the United Kingdom. Age Ageing 2009;38:27-32.

10. Merriman NA, Sexton E, Donnelly NA, et al. Managing cognitive impairment following stroke: protocol for a systematic review of nonrandomised controlled studies of psychological interventions. BMJ Open 2018;8:e019001.

11. Cicerone KD, Dahlberg C, Malec JF, et al. Evidence-based cognitive rehabilitation: updated review of the literature from 1998 through 2002. Arch Phys Med Rehabil 2005;86:1681-92.

12. Hoffmann T, Bennett S, Koh CL, et al. Occupational therapy for cognitive impairment in stroke patients. Cochrane Database Syst Rev 2010;9:CD006430.

13. das Nair $\mathrm{R}$, Cogger $\mathrm{H}$, Worthington $\mathrm{E}$, et al. Cognitive rehabilitation for memory deficits after stroke. Cochrane Database Syst Rev 2016;9:CD002293.

14. Chung CS, Pollock A, Campbell T, et al. Cognitive rehabilitation for executive dysfunction in adults with stroke or other adult nonprogressive acquired brain damage. Cochrane Database Syst Rev 2013:CD008391.

15. Bowen A, Hazelton C, Pollock A, et al. Cognitive rehabilitation for spatial neglect following stroke. Cochrane Database Syst Rev 2013;7:CD003586.

16. Loetscher T, Lincoln NB. Cognitive rehabilitation for attention deficits following stroke. Cochrane Database Syst Rev 2013;5:CD002842.

17. Renton T, Tibbles A, Topolovec-Vranic J. Neurofeedback as a form of cognitive rehabilitation therapy following stroke: A systematic review. PLoS One 2017;12:e0177290.

18. Sachdev PS, Brodaty $\mathrm{H}$, Valenzuela MJ, et al. The neuropsychological profile of vascular cognitive impairment in stroke and TIA patients. Neurology 2004;62:912-9.

19. Vasquez BP, Zakzanis KK. The neuropsychological profile of vascular cognitive impairment not demented: a meta-analysis. J Neuropsychol 2015:9:109-36

20. Barker-Collo S, Starkey N, Lawes CM, et al. Neuropsychological profiles of 5-year ischemic stroke survivors by Oxfordshire stroke classification and hemisphere of lesion. Stroke 2012;43:50-5.

21. Nys GM, Van Zandvoort MJ, De Kort PL, et al. Domain-specific cognitive recovery after first-ever stroke: a follow-up study of 111 cases. J Int Neuropsychol Soc 2005;11:795-806.

22. Moher D, Liberati A, Tetzlaff J, et al. Preferred reporting items for systematic reviews and meta-analyses: the PRISMA statement. Ann Intern Med 2009;151:264-9.

23. Liberati A, Altman DG, Tetzlaff J, et al. The PRISMA statement for reporting systematic reviews and meta-analyses of studies that evaluate health care interventions: explanation and elaboration. PLoS Med 2009;6:e1000100

24. Hachinski V, ladecola C, Petersen RC, et al. National Institute of Neurological Disorders and Stroke-Canadian Stroke Network vascular cognitive impairment harmonization standards. Stroke 2006;37:2220-41.

25. Nasreddine ZS, Phillips NA, Bédirian V, et al. The Montreal Cognitive Assessment, MoCA: a brief screening tool for mild cognitive impairment. J Am Geriatr Soc 2005;53:695-9.

26. Folstein MF, Folstein SE, McHugh PR. "Mini-mental state". A practical method for grading the cognitive state of patients for the clinician. J Psychiatr Res 1975;12:189-98.

27. Katz N, Itzkovich M, Averbuch S, et al. Loewenstein Occupational Therapy Cognitive Assessment (LOTCA) battery for brain-injured patients: reliability and validity. Am J Occup Ther 1989;43:184-92.

28. Doninger NA, Ehde DM, Bode RK, et al. Measurement properties of the neurobehavioral cognitive status examination (Cognistat) in traumatic brain injury rehabilitation. Rehabil Psychol 2006;51:281-8.

29. Troyer AK, Rich JB. Psychometric properties of a new metamemory questionnaire for older adults. J Gerontol B Psychol Sci Soc Sci 2002;57:P19-27.

30. Keith RA, Granger CV, Hamilton BB, et al. The functional independence measure: a new tool for rehabilitation. Adv Clin Rehabil 1987;1:6-18.

31. Nouri FM, Lincoln NB. An extended activities of daily living scale for stroke patients. Clin Rehabil 1987;1:301-5.

32. Hozo SP, Djulbegovic B, Hozo I. Estimating the mean and variance from the median, range, and the size of a sample. BMC Med Res Methodol 2005:5:1-10. 
33. Miller LA, Radford K. Testing the effectiveness of group-based memory rehabilitation in chronic stroke patients. Neuropsychol Rehabil 2014;24:721-37.

34. Young GC, Collins D, Hren M. Effect of pairing scanning training with block design training in the remediation of perceptual problems in left hemiplegics. J Clin Neuropsychol 1983;5:201-12.

35. Higgins JP, Green S, eds. Cochrane Handbook for Systematic Reviews of Interventions Version 5.1.0: The Cochrane Collaboration, 2011. www.cochrane-handbook.org

36. Sterne JA, Hernán MA, Reeves BC, et al. ROBINS-I: a tool for assessing risk of bias in non-randomised studies of interventions. BMJ 2016;355:i4919.

37. Sterne JA, Sutton AJ, loannidis JP, et al. Recommendations for examining and interpreting funnel plot asymmetry in meta-analyses of randomised controlled trials. BMJ 2011;343:d4002-8.

38. Guyatt GH, Oxman AD, Vist GE, et al. GRADE: an emerging consensus on rating quality of evidence and strength of recommendations. BMJ 2008;336:924-6.

39. Hedges LV, Distribution theory for glass's estimator of effect size and related estimators. Journal of Educational Statistics 1981;6:107-28.

40. Becker BJ. Synthesizing standardized mean-change measures. $\mathrm{Br} \mathrm{J}$ Math Stat Psychol 1988;41:257-78.

41. Cohen J. Statistical power analysis for the behavioural sciences. rev. ed. New York, NY: Academic Press, 1977.

42. Scammacca N, Roberts G, Stuebing KK. Meta-analysis with complex research designs: dealing with dependence from multiple measures and multiple group comparisons. Rev Educ Res 2014;84:328-64.

43. Hill NT, Mowszowski L, Naismith SL, et al. Computerized cognitive training in older adults with mild cognitive impairment or dementia: a systematic review and meta-analysis. Am J Psychiatry 2017:174:329-40.

44. Lampit A, Hallock $\mathrm{H}$, Valenzuela M. Computerized cognitive training in cognitively healthy older adults: a systematic review and metaanalysis of effect modifiers. PLoS Med 2014;11:e1001756.

45. Higgins JP, Thompson SG, Deeks JJ, et al. Measuring inconsistency in meta-analyses. BMJ 2003;327:557-60.

46. Poulin V, Korner-Bitensky N, Bherer L, et al. Comparison of two cognitive interventions for adults experiencing executive dysfunction post-stroke: a pilot study. Disabil Rehabil 2017;39:1-13.

47. Sturm W, Willmes K, Orgass B, et al. Do specific attention deficits need specific training? Neuropsychol Rehabil 1997;7:81-103.

48. Sturm W, Longoni $F$, Weis $S$, et al. Functional reorganisation in patients with right hemisphere stroke after training of alertness: a longitudinal PET and fMRI study in eight cases. Neuropsychologia 2004:42:434-50.

49. Hildebrandt H, Bussmann-Mork B, Schwendemann G. Group therapy for memory impaired patients: a partial remediation is possible. J Neurol 2006;253:512-9.

50. Lindeløv JK, Dall JO, Kristensen CD, et al. Training and transfer effects of $\mathrm{N}$-back training for brain-injured and healthy subjects. Neuropsychol Rehabil 2016;26:895-909.

51. Gauggel S, Niemann T. Evaluation of a short-term computer-assisted training programme for the remediation of attentional deficits after brain injury: a preliminary study. Int $J$ Rehabil Res 1996;19:229-40.

52. Mitrovic A, Mathews M, Ohlsson S, et al. Computer-based poststroke rehabilitation of prospective memory. J Appl Res Mem Cogn 2016;5:204-14.
53. Honda T. Rehabilitation of executive function impairments after stroke. Top Stroke Rehabil 1999;6:15-22.

54. Towle D, Edmans JA, Lincoln NB. Use of computer-presented games with memory-impaired stroke patients. Clin Rehabil 1988;2:303-7.

55. Li K, Robertson J, Ramos J, et al. Computer-based cognitive retraining for adults with chronic acquired brain injury: a pilot study. Occup Ther Health Care 2013;27:333-44.

56. Yip BC, Man DW. Virtual reality (VR)-based community living skills training for people with acquired brain injury: A pilot study. Brain Inj 2009;23:1017-26.

57. Ressner P, Niliu P. Computer-assisted cognitive rehabilitation in stroke and Alzheimer?s disease. J Neurol Neurophysiol 2014:05:4-7.

58. Thomas KS, Hicks JJ, Johnson OA. A Pilot Project for Group Cognitive Retraining with Elderly Stroke Patients. Phys Occup Ther Geriatr 1995;12:51-66.

59. Stamenova V, Jennings JM, Cook SP, et al. Repetition-lag memory training is feasible in patients with chronic stroke, including those with memory problems. Brain Inj 2017;31:57-67.

60. Łojek E, Bolewska A. The effectiveness of computer-assisted cognitive rehabilitation in brain-damaged patients. Polish Psychological Bulletin 2013;44:31-9.

61. Piccardi L, Nico D, Bureca I, et al. Efficacy of visuo-spatial training in right-brain damaged patients with spatial hemineglect and attention disorders. Cortex 2006;42:973-82.

62. Liu KP, Chan CC, Lee TM, et al. Mental imagery for relearning of people after brain injury. Brain Inj 2004;18:1163-72.

63. Pyun SB, Yang H, Lee S, et al. A home programme for patients with cognitive dysfunction: a pilot study. Brain Inj 2009;23:686-92.

64. Hayes S, Donnellan C, Stokes E. Executive dysfunction post-stroke: an insight into the perspectives of physiotherapists. Disabil Rehabil 2015;37:1817-24.

65. Van Heugten CM, Walton L, Hentschel U. Can we forget the MiniMental State Examination? A systematic review of the validity of cognitive screening instruments within one month after stroke. Clin Rehabil 2015;29:694-704.

66. Balk EM, Bonis PA, Moskowitz $\mathrm{H}$, et al. Correlation of quality measures with estimates of treatment effect in meta-analyses of randomized controlled trials. JAMA 2002;287:2973-82.

67. Poulin V, Korner-Bitensky N, Dawson DR, et al. Efficacy of executive function interventions after stroke: a systematic review. Top Stroke Rehabil 2012;19:158-71.

68. Pohjasvaara T, Leskelä M, Vataja R, et al. Post-stroke depression, executive dysfunction and functional outcome. Eur J Neurol 2002;9:269-75

69. Román GC, Royall DR. Executive control function: a rational basis for the diagnosis of vascular dementia. Alzheimer Dis Assoc Disord 1999;13(Suppl3):S69-80.

70. Hoffmann TC, Glasziou PP, Boutron I, et al. Better reporting of interventions: template for intervention description and replication (TIDieR) checklist and guide. BMJ 2014;348:g1687.

71. The StrokeCog study: modelling and modifying the consequences of stroke-related cognitive impairment through intervention [Internet]. http://www.hrb.ie/research-strategy-funding/grants-and-fellowships/ funding-awarded/funding-award/awards//639/ 